\title{
Mobile Internet Travel Cloud Computing Data Prediction Algorithm Research
}

\author{
Lianlei Fan and Shuguang Fu \\ Qingdao Vocational and Technical College of Hotel Management \\ Fanll423@163.com,Zsbqchm@126.com
}

\begin{abstract}
Mobile Internet make human whenever and wherever access to the Internet possibly, achieving online booking, according to the mobile phone location data, we can acquire the relevant data tourist attractions, through the real data of tourist arrivals, traffic load data, according to the computing resources of mobile Internet, and the data on the travel data mining algorithms to predict, in order to achieve tourism management; this article aim at mobile Internet cloud computing resource allocation and data mining algorithms to predict data, the simulation results can be obtained, the accuracy of the data is more stronger.
\end{abstract}

Keywords: Mobile Internet; cloud computing; data mining; tourism prediction

\section{Introduction}

Mobile Internet has become the main development structure of the Internet, because the mobile terminal can access to Internet anytime, anywhere, to become the mainstream now with the topology of network application. Because cloud computing can decompose large amounts of data into multiple computing terminal for processing, so as to realize the parallel processing of data, data processing and data mining is widely used in the Internet; however, due to the mobile Internet is a newly emerging technology, the mobile Internet cloud computing method still in the initial stage of the study. At the same time, in the acquisition of related tourism service, due to the emergence of mobile Internet, tourists can through the mobile terminal, especially intelligent mobile phone, to private custom tourism related information they need, such as the route chart, according to the network, tourism related spots fares can use exchange system, and selected platform for online booking service [1]. So, how to build a suitable mobile internet tourism information platform, and based on the data of this platform and the operation custom of users to data mining and analyze big data on the network platform. In addition to data analysis, it can also predict future data of tourism based on the past historical data records [2]. 


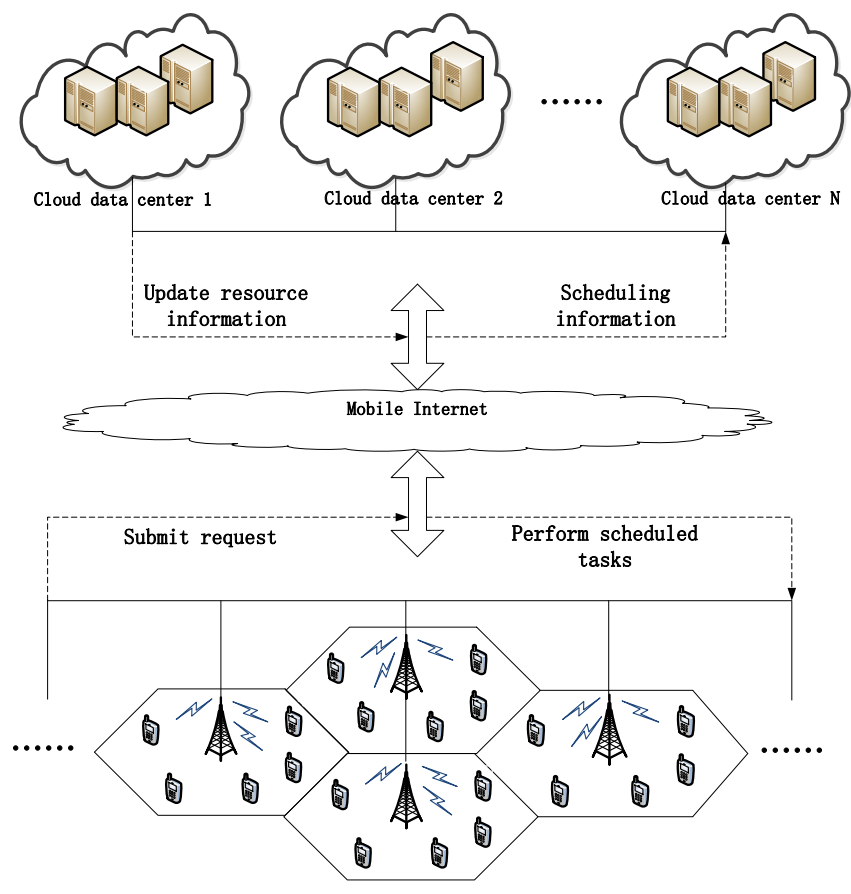

Figure 1. Mobile Internet Topology Map

Traditional data mining algorithms and methods can not fit massive data analysis and forecasting, and the traditional cloud computing method, although it can compute and make data mining for massive data, but it cannot adapt to the mobile Internet whenever access to. Hence, it needs to design the characteristics of mobile Internet cloud computing methods to meet the travel data structure. With the development of computing and communication capabilities of mobile devices, as well as storage capacity has been greatly improved, such as smart phone, laptops and tablet computers. Statistics show that, by 2015 , mobile devices will outnumber wired devices, becoming a major equipment to access the Internet. This effect changes the access from the terminal to the problems posed by the distributed computing resource pool, the mobile access network and the core of the Internet composed of the mobile Internet computing resources and storage resources and the number of nodes and different from the traditional Internet, thus it requires a different way of resource scheduling, and with the business applications of smart phones and tablet computers and other equipment, there are many sensing devices and sensor data, such as gravity, direction and GPS location information can provide distributed information space, giving resource scheduling brings convenient. Meanwhile, cloud computing may cause the mobile Internet to obtain the following three resources: First, getting the storage resources of the network; second, obtaining computing resources of the network; third, obtaining application service resources of the other network access node.

While cloud computing used for computing and the deployment of the storage resources, but the traditional Internet cloud computing does not consider the issue of energy consumption and bandwidth problems, so the traditional approach to cloud computing on the Internet cannot be directly applied to the mobile Internet environment. Cloud computing and mobile Internet get more and more academic attention.

Mobile Internet mainly in mobile devices wireless access to, the energy provider is limited, in the fifth generation mobile communication systems, the concept of 
green communication is popular, but its design is data transmission of the physical layer and resource scheduling of the data link layer, it mainly focus on the data transmission within the network; however, through the application layer, rational management of computing and storage resources of mobile Internet terminal also have few involved studies .Informatization brings revolutionary change again and again for development and enhancement of the tourism. From the point of view of tourism services platform, hotels, airlines, travel agencies and other travel service providers make their initial line of business moved to the Internet, forming a single tourism service platform, followed by food, lodging, transportation, travel, shopping, entertainment, etc. one-stop service platform at home and abroad become very popular, which are dependent on network technology support. Advances in technology, making the experience of information service and delivery get better, tourism service providers also won the lucrative returns, while the tourism industry but also to the economic development of the country has made a great contribution to the tourism industry with the rapid development of information technology and tourism are inseparable, the close relationship between the key to survival of the fittest in the high-tech industry, which has become an important tool to strengthen tourism competitiveness and methods. Thus the high-tech and tourism related services, advanced platform and philosophy has been changed, the previous simple service delivery platform, now being quietly transition [3-5].

In particular, the popularity of mobile intelligent terminal, making social networks flourish, with social networking platforms such as Microblog, Wechat, qq and other platforms are stationed travel services provider, and according to the different needs of tourism, many sites were also established a generation buy train tickets, tickets, accommodation and other recommended services, such as Ctrip network. However, in the vast data and platforms, how can we construct a line with individual needs, and has diversified and personalized private order, which requires the data mining and decision-making, in order to make relevant alternatives, and the need to give advice, this proposal comes from the future traffic and other tourist services at various points of data to predict. Among the Internet has like lvmama, tuniu Web sites carry a similar service, but this distance to personalized service, Personal Tailor has been optimized and other targets are still far away, and its source data is based on a fixed data, far from reflecting the real tourist situation, based on the current situation and needs of the mobile Internet, according to data characteristics and topological structure, this article designs a cloud computing platform to fit the characteristics of tourism data, based on the arrival characteristics and structural features of the data, it able to give relevant travel advice.

\section{Related Work}

\subsection{Mobile Internet Cloud Computing Research Status}

[6] uses a continuous-time Lagrangian duality scheduling principle to schedules data resources, and its optimization objective function is minimized energy consumption. [5] studied the energy efficiency of online awareness and delay transmission structures. [7] considers control algorithm based on energy efficiency of the varying channel conditions. However, the conditions mentioned above algorithm design is too ideal, they considered a user to transfer data.

Then [8], in a base station, in $\mathrm{N}$ time-varying channels provide services for $\mathrm{N}$ users, and proposed an adaptive algorithm of channel acquisition. [9] proposed an adaptive carrier aggregation approach to change the energy efficiency of data download and upload. As can be seen, the current study is based on the combination 
between the physical layer transport and the application layer scheduling, the focus is still on the physical layer, the application layer services are mainly concentrated in the upload and download of such data transmission, which is obviously does not meet the requirements of the mobile Internet. [10] studied the work of computational model of the Internet, which according to the method of cloud computing in the online dynamic allocation of computing the amount of the accord. In order to improve the efficiency of distribution, [11] used a competitive bidding to allocate computing workloads out. This approach simplifies the computational complexity of the allocated algorithm, but the distribution effect is not as good as other high computational complexity algorithm. On this basis, [12] proposed a resources allocation that joint optimization of demand and allocation, this mode can quickly allocate resources, and lower computational complexity. However, more of these algorithms cannot be applied to the mobile Internet, due to computational capability of a single node is lower, and the communication capability between each node are lower than the Internet. Hence, it needs to find a scheduling assignment algorithm to suit for mobile Internet. Through studying estimated model of work load, [12] based on distributed online collection algorithm presents a VM dynamic supply strategy. In order to improve work efficiency of the cloud computing, [13] put forward a computing resource auction mechanism which based on MapReduce, this mechanism can take appropriate computational complexity to obtain higher work efficiency, and however, these two methods are needed to predict the work load. Therefore, literature [8] presented a VM dynamic scheduling mechanisms that it does not need to predict work load, the mechanism only according to the current user demand for computing resources through the auction to configure and offer VM to the user. The biggest drawback of using the auction mechanism for resource scheduling is the high computational complexity, in order to reduce the computational complexity, the literature [9] according to the real auction mechanism proposes a combined strategy of dynamic allocation and supply VM auction based on this strategy to make a judgment according to the user request VM real bid value, the user only actual bid to ensure the maximum benefit, this strategy has the advantage of auction judgment does not need the previous auction information, it can effectively reduce the computational complexity. However, these methods design which are based on the data center, without considering the limitation of wireless network bandwidth, although it can be used for mobile cloud computing scene, but it also faces some problems. For example: when the data center to the super busy area in the allocation of VM after a great number of users, leading to the cell bandwidth restrictions make the system cannot timely service data to be delivered to the user happens, not only affects the mobile cloud computing service quality, but also the impact of the cloud provider's benefit. Thus, limited bandwidth problem is shouldn't be ignored for VM scheduling problem when mobile cloud computing.

\subsection{Data Framework of Tourism Cloud Platform}

The proposal of cloud computing and advance of tourism commercial, promoting tourism information, intelligence and the custom of tourism. So gradually the proposed "tourism cloud" in 2010, the concept of cloud computing promote the development of tourism cloud, but tourism is based on the traditional Internet cloud framework, real-time data and the diversity of its operations cannot compare to mobile Internet. Proposed cloud computing needs to be extended to all areas of the application, where tourism is a very important area. Cloud computing is currently mainly IaaS, PaaS and SaaS model rendering services, and the associated types of $\mathrm{XaaS}$ (X represents at least greater than 3) mode is defined. Meanwhile, the 
application model in order to define cloud computing, cloud computing is usually divided into public clouds, private clouds and hybrid clouds, etc. According to the International Data Corporation statistics show that the cloud in just a few years, it has been applied in various fields, and produced a huge economic value. Currently, the well-known cloud computing platforms like Google Apps Engine, Amazon EC2, Microsoft office live, NetSuite Suiteflex, IBM Blue Cloud, etc., and specialized applications in the field of cloud are: cloud manufacturing, cloud simulation platform, Grid cloud platform, efficient scientific computing cloud, cloud-based education platform. People in academia also launched a wealth of cloud computing research, clouds trust / security studies, resource assessment modeling and simulation, cloud computing and cloud services quality architecture composed of cloud environments, etc. In [14], the authors analyze and summarize the concepts and overview of cloud computing platforms, from the perspective of the concept and platform to sort out in the end is what cloud computing; in the literature [15], the authors reviewed the system architecture of cloud computing the latest research progress and critical computing technologies and cloud; and in the literature [16], a more comprehensive overview of the theory and research status examples of cloud computing. In tourism cloud, IaaS framework which can analyze the distribution of computing features include configuration capabilities, population characteristics of tourism resources of tourism and its user data. It constitutes the entire tourism infrastructure cloud; in PaaS architecture can be extended based IaaS framework, adaptability and ability obviously been expanded, you can build a variety of application platform based on it. On the basis of the two frames, we can build SaaS framework to further optimize business system that can target different needs, that is, different data structures, which can be efficiently and accurately custom software.

Tourist cloud uses cloud computing principles and techniques, as well as a tour to meet the needs of the parties to the framework structure tourism information Internet-based distributed computing model to build, and can be carried out in accordance with all requirements on the cloud platform to build tourism dynamic deployment, configuration, reconfiguration, and cancellation of the new tourist information model; but also to meet the high demand cloud computing reusability, scalability computing, load balancing, and other characteristics can be rented. Parties should include tourism resource virtualization, and these resources are virtualized tourism is a safe and reliable application and reuse; usually virtualized resources may include server virtualization, storage virtualization is intended, application virtualization platform virtualization and desktop virtualization. 1) Server Virtualization: it is to make one or several physical server into several virtual complete, trusted server to use for different needs; it is also the basis of IaaS, providing you can use / server support for different rental needs. 2) Storage Virtualization: It is the entire tourism cloud storage resources unified integrated management system to provide a unified for different user needs, independent of storage space and a unified optimization, collaborative management of the tourism cloud data storage; 3) Application Virtualization: it is to travel cloud each cloud ( different user groups ) applications rely on the underlying system and hardware abstraction, forming an independent and integrated applications to decouple applications and operating systems, hardware relationship. Tourism Management cloud: mainly by the administrative department of tourism, tourist management department composition, customized to the features of the two kinds of different management interface management. The Department of tourism management can be divided into provincial, state, county (city) level and tourism management level four customizable interface management, provincial can the overall management of the province's tourism resources, state (city) level management department is the macro 
grasp of the tourism resources in all state(city), county under the jurisdiction of the activities only manages all county tourism resources activities, tourism management level only manages the territorial tourism activity changes. And the management information dynamically customized according to local tourism rules and related policies, in order to achieve each management link and orderly, and can by the human nature management strategy to serve the local economic construction, and to provide the most affordable tourism marketing plan for travel consumer users, to achieve the rational use of tourism resources. Passenger cloud: mainly refers to the clouds from different regions of the tourism consumers, their main goal are to obtain satisfy tourist consumption planning their own needs. This cloud has close relationship with the tourism management cloud, scenic area cloud and tourism partners cloud; their service level, the tourism resource development, marketing strategy directly affect the tourism consumers' choice, and further influence the development of regional tourism economy. At the same time, service level, service ability of these clouds directly influence on tourism customer satisfaction and loyalty of the region's tourism. Meanwhile, the tourism managers, it can also change the management strategy and marketing strategy according to the characteristics of passenger. The traditional calculation method is very difficult to do this, the emergence of cloud computing has created the condition for the improvement or change of tourism dynamic management, dynamic customization and dynamic maintenance is possible. For the client to cloud computing data mining algorithm, this article can get the information and advice of tourism dynamic on mobile terminal, it also can travel private custom whenever and wherever possible business.

\section{Proposed Scheme}

According to the characteristics of data mining algorithm, and aiming at the characteristics of mobile Internet the topological structure, improving the traditional association rules, forming a new fast convergence algorithm, this algorithm can integrate a variety of needs, according to the sequence of the size and needs of relevance between each demand; it can quickly get Association data. In the association rules, the most classic algorithm is Apriori. The subsequent association rules algorithm is also based on this idea, and improved it.

\subsection{The Data Mining Method based on the Proportion of Incremental Improvement}

A collection of DB data quantity is set $D$, the size of new data services set $d B$ is $D$, and the new business data collection is far less than the original set of data business, frequent item set storage and data set is obtained in the DB class for L (D). According to the following method of data association to measure and update. The first step for business data, new sets of association rules discovery, thus getting the frequent business and the corresponding set of L (d), based on improved Apriori algorithm. Obtaining the frequent operations of the new collection of L (d), its frequent business and the business of the original data set L (D) were compared, finding the intersection of the two sets, and the intersection with the original business data collection and calculation of part; The second step, the first step obtained the set of data after computing is divided into P subsets of data, and the collection of various subsets of data and the candidate are sent to $\mathrm{P}$ virtual machine; The third step, the virtual machine and a subset of data set and candidate data comparison, finding the matching set; Fourth step, according to distinguish measure function, doing the distinction degree measure of match data, calculating the total 
distinction degree; The fifth step, according to the discrimination and limits the total, screening out the candidate data set according to the options, options can be frequent business screened set; The sixth step, according to the set of frequent business, respectively to each virtual machine, virtual machine according to the frequent business element data update, obtain the optimal business element options, will send a service option to the general decision making machine; The seventh step, the general decision optimal option machine according to each virtual machine to the proposed integrated set, frequent business elements of the proposed options.

Frequent business data described by the $\mathrm{n}$ property, each data represents a point in $\mathrm{n}$ dimensional space. By this method, all the training tuples are stored in the model of in $\mathrm{n}$ dimension space. When given an unknown tuple space, model $\mathrm{k}$ training tuple $\mathrm{k}$ - nearest neighbor classifier to search the most close to the unknown tuple. These $\mathrm{k}$ training tuples is unknown tuple $\mathrm{k}$ "nearest neighbor". "Close to" the definition is distance metrics, such as the Euclidean distance or similarity. Two Euclidean distance between points or tuples, the Euclidean distance between two points can be expressed as

$$
D\left(\mathrm{X}_{1}, \mathrm{X}_{2}\right)=\sqrt{\sum_{i=1}^{n}\left(x_{1 i}-x_{2 i}\right)^{2}}
$$

where, the algorithm is suitable for numerical data type, and for the discrete data, using two vector multiplication sum mode, so it can maximize its different data were weighted sum. For vector data, the angle between vectors can be the cosine measure. It is calculated as

$$
\cos \left(\mathrm{X}_{1}, \mathrm{X}_{2}\right)=\sum_{i=1}^{n} x_{1 i} \times x_{2 i} / \sqrt{\sum_{i=1}^{n} x_{1 i}^{2}} \sqrt{\sum_{i=1}^{n} x_{2 i}^{2}}
$$

If using this formula on the serial machine, the complexity of time is linear close to the number of data node. Therefore, it needs to calculate way mapped to a highly parallel computer process, and further optimizing operation time of algorithm. The above is for solving methods, difference distinction can be seen, the low computational complexity, which ensure the real-time operation, and because the operation in multiple parallel virtual machine, the time is reduced by $\mathrm{K}$ times, it can get the mobile Internet cloud computing. And each virtual machine processing of data is not large, which can act as a virtual machine for mobile terminals, such as virtual machine many data, so that data have credibility, and the terminal data is real-time, so the update is very timely, suitable for carrying out tourism business.

\subsection{Computing Resource Scheduling based on Energy Efficiency}

In the mobile internet, given $n$ business types require scheduling, because there is a lot of the mobile Internet business types, so the value of $n$ is large, the provided business are $S_{1}, \mathrm{~L} S_{n}$, And the computational resources required for each type of business is $r_{i}$, in this article, using $r=\left(r_{1}, r_{2}, \cdots, r_{n}\right)$ represents $n$ different business computing resources. Setting mobile Internet computing resources can be mobilized into $n$ classes, each computing function can be represented as $c_{i}, c_{1}$ is the normalization of computing function, so $c_{1}=1$; we can use $\mathbf{C}=\left(c_{1}, c_{2}, \cdots, c_{m}\right)$ denotes the calculation of $m$ virtual machine, due to $c_{1}$ is normalized, so the vector $\mathbf{c}$ can be regarded as the ratio of the number of computing power. For example, if the cloud computing service provider of mobile Internet can provide the virtual machine which has 4 kinds computing capacity, they are $c_{1} 、 c_{2} 、 c_{3}$ and $c_{4}$; where computing resources that can be mobilized of $c_{1}$ is $1 \mathrm{GHz}$ processing dominant frequency, 2GB 
call memory and 500GB hard disk; The computing resources of $c_{2}$ is dual-core 1 GHZ processing dominant frequency, 4GB call memory and 1TB hard disk; The computing resources of $c_{3}$ is quad-core $1 \mathrm{GHZ}$ processing dominant frequency, 8GB call memory and $2 \mathrm{~TB}$ hard disk; $c_{4}$ is octa-core $1 \mathrm{GHZ}$ processing dominant frequency, 16GB call memory and $4 \mathrm{~TB}$ hard disk, thus the consisting of a vector for computing resources ability is $\mathbf{C}=(1,2,3,4)$. If the number of mobile Internet services for cloud computing can provide that the $i$-th class for $l_{i}$, the vector that was composed of the number provided by various types virtual machines is $\mathbf{L}=\left(l_{1}, l_{2}, \cdots, l_{m}\right)^{T}$, it should meet the conditions for

$\mathbf{C} \mathbf{L} \leq M$

where, ${ }_{m}$ represents that the total number of can provide all kinds of calculate business. In this section, $T=\left\{t_{1}, t_{2}, \cdots, t_{n}\right\}$ is the set of all the tasks that needed to deal with; considering the data which requires processing doing data exchange between $t_{i}$ and $t_{j}$, the exchanged data is $d_{i j}$, the maximum mutual information of the channel

can be expressed as $i_{i j}$, the computing power of $i$-th virtual machines as $p_{i}$, time required for the completion of this task can be represented by the Formula (4)

$$
T_{l_{i}}=d_{i j} /\left(P_{i} * \rho_{l_{i}}\right)
$$

where, $\rho_{t_{1}}$ represents the computing power of this task from node on. However, due to bandwidth limitations, it needs to consider the transmission time of the node transmit information, setting for transmission time as $T_{c_{l}}$ (including transmission time before and after processing), therefore, the total time for doing this business is $T_{z_{i}}$. According to the virtual machine to provide computing services, supposing consumed energy for computing services of each virtual machine unit is $E_{t_{l}}$, due to different propagation systems, according to the network system, which needs to transfer the data required for the unit (including the required energy for the uplink and downlink data ) is $E_{c \mid}$, Which we can obtain the energy that transfer needed for

$$
E_{z l_{i}}=E_{c l_{i}} d_{i j}+E_{l_{i}} t_{1}
$$

According to the above definition of the model, allocation of resources may be needed to define, in the conventional optimization model, often emphasis processing service time, that the business processing delay, which is also an important indicator of the fifth generation of mobile cellular communications, but there is an important indicator for handling operations required energy consumption for energy issues, we propose to optimize energy consumption and processing delay problems into account, it raised the following optimization problem

$$
\begin{aligned}
& \mathrm{m} \text { in } E_{z} \\
& \text { s.t. } T_{z_{i}} \leq \eta_{i}
\end{aligned}
$$

where, $E_{z}$ is the energy for all tasks, it can be represented by (7) 


$$
E_{\mathrm{Z}}=\sum_{i=1}^{M} E_{i_{i}}
$$

In the actual search process, due to the heterogeneous network, the user changes, resulting in changes in the virtual machine, the energy of each task becomes consumed in transmitting data is changed, therefore, the speed of computational resources is also scheduled to be considered the key issues. In this article, we selected PSO algorithm, this algorithm can ensure that the search for the solution has the fastest convergence rate, and the way to obtain execution of parallel computing, but because the particle swarm algorithm to search for the solution is easy to fall into local optimal solution, thus affecting the effectiveness of resource scheduling, therefore, this paper PSO modified to ensure the solution has the characteristics of the search of the optimal solution, and to ensure the convergence speed.

First, determining the energy particle for consumption of task, but for the search direction of energy consumption, this paper adopts is the gradient progressive method, namely the search direction according to the partial derivative in the direction of a search, i.e.

$$
\sigma=\frac{\partial E_{z}}{\partial E_{l_{i}}}
$$

But the point of its search for the end of last search, most initialization of the starting point, selecting the calculated initialization energy consumption as a search starting point. It can search in the following form:

$$
\begin{aligned}
E_{i}(n+1)= & E_{i}(n)+\mathbf{v}_{i}(n) \\
\mathbf{v}_{i}(n+1)= & \sigma \mathbf{v}_{i}(n)+\lambda_{1} \theta_{1}\left(E_{i}^{*}(n)-E_{i}(n)\right) \\
& +\lambda_{2} \theta_{2}\left(E^{*}(n)-E_{i}(n)\right)
\end{aligned}
$$

where, $\sigma$ represents the convergence rate proportion from the last step search; $\lambda_{1}$ and $\lambda_{2}$ are proportion vectors of two conditions, this vector was used to search the required direction, the combination is the two-dimensional direction adjustment. $\beta_{1}$ and $\beta_{2}$ are acceleration factors, $E_{i}^{*}(n)$ is the optimal solution of this search, $E_{i}(n)$ is the specific position of this search.

\section{Experimental Results and Analysis}

In this paper, the case due to cloud computing and simulation models for the mobile Internet, they need to build a cloud simulation platform, this cloud computing platform cloud model simulations common platform CloudSim, it evolved from its foundation on distributed and parallel computing using this platform can store computer simulation resources and transfer of data, but the lack of link topology changes, according to this reality, this experiment has been modified for this, this modified according to the topology diagram to modify the transmission of data and transmission time. Simulation environment, including computer configuration environment. Computer simulation of its environment are shown in Table 1. 
Table 1. VM Configuration

\begin{tabular}{cccc}
\hline & processor & memory & hard disk \\
\hline$V M_{1}$ & $1 \times 2$ & $4 \mathrm{GHz}$ & $500 \mathrm{~GB}$ \\
& $\mathrm{GHz}$ & & \\
$V M_{2}$ & $2 \times 2$ & $8 \mathrm{GHz}$ & $1 \mathrm{~TB}$ \\
& $\mathrm{GHz}$ & & \\
$V M_{3}$ & $4 \times 2$ & $16 \mathrm{GHz}$ & $2 \mathrm{~TB}$ \\
& $\mathrm{GHz}$ & & \\
$V M_{4}$ & $8 \times 2$ & $32 \mathrm{GHz}$ & $4 \mathrm{~TB}$ \\
\hline
\end{tabular}

The journey is usually the simulation as shown below, according to the virtual task and scheduling implementation model for the development of its core algorithm written, its scheduling intervals depending on the simulation environment, the need for a separate set.

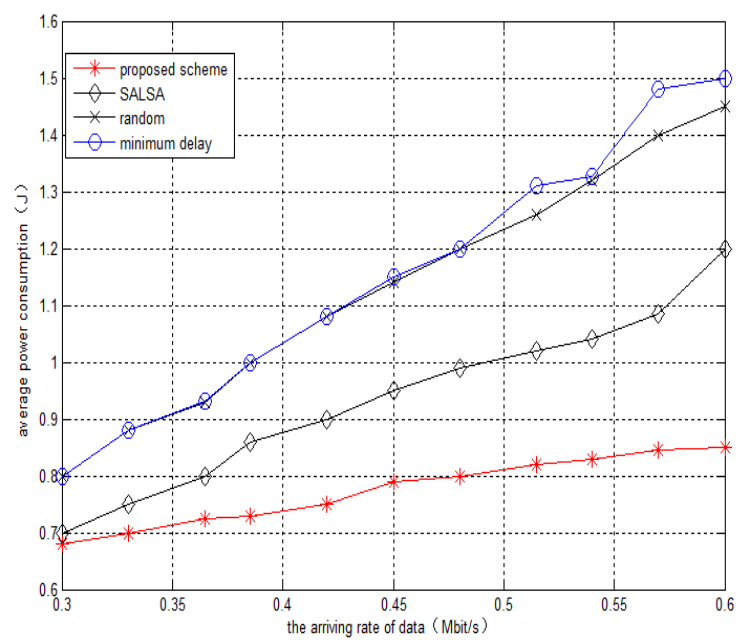

Figure 2. Comparison of Average Energy Consumption

Figure 2, shows that the improved scheme proposed in this paper, the advantages in terms of energy utilization, i.e. according to different topologies and execution time, costs is lower than with traditional PSO program. And with the growth of data arrival rate, the more obvious advantages. As can be seen, because of the tourism business reach a wider distribution of their data, that is, over time, there will be a burst of data arrives situation, but the lowest energy consumption in the entire data showed the arrival rate, with a strong robustness. Thus saving power of the mobile terminal. 


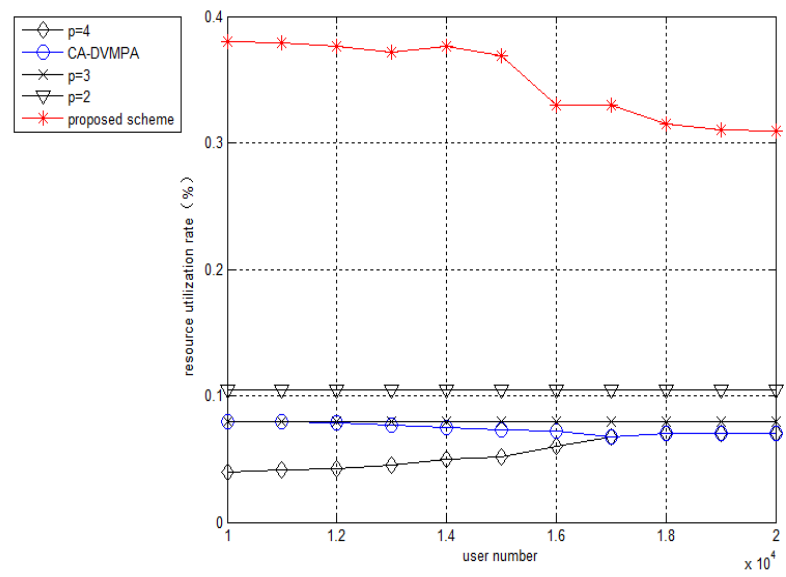

Figure 3. Comparison of Resource Utilization Rate

The resource utilization of Figure 3, can be seen, resource utilization of the proposed algorithm is significantly higher than other programs, we can see that the proposed scheme can have a strong computing power in business; thus it can be done fast data mining tourist information, resulting in a mobile cloud platform tourist information advice efficiency is high. As can be seen, the higher the computational efficiency of the proposed tourist mobile cloud platform is even higher.

\section{Conclusion}

According to the characteristics and tourism information of mobile Internet, constructing the screening method of data, using the weighted proportion Apriori algorithm; in the calculation of resource allocation, this paper optimized the energy efficiency programs, and took the business processing time into account, through the simulation platform experiment, the performance of energy and resource utilization rate have been greatly improved.

\section{References}

[1] R. M. Wagner, W. Jing and Y. Han, "Requirement identification and experiments for a flexible media format based on mobile and cloud computing", Information Networking (ICOIN), 2014 International Conference on IEEE, (2014), pp. 202-207.

[2] H. Gangwar, H. Date and R. Ramaswamy, "Understanding determinants of cloud computing adoption using an integrated TAM-TOE model", Journal of Enterprise Information Management, vol. 28, no. 1, (2015).

[3] C. Y. Tang, C. C. Lai and C. W. Law, "Examining key determinants of mobile wallet adoption intention in Malaysia: an empirical study using the unified theory of acceptance and use of technology 2 model",International Journal of Modelling in Operations Management, vol. 4, no. 3, (2014), pp. 248-265.

[4] P. J. Lin, C. C. Kao and K. H. Lam, "Design and Implementation of a Tourism System Using Mobile Augmented Reality and GIS Technologies", Proceedings of the 2nd International Conference on Intelligent Technologies and Engineering Systems (ICITES2013), Springer International Publishing, (2014), pp. 1093-1099.

[5] M. A. Nour, "An Empirical Study of the Effect of Internet Services on the Preferential Adoption of Mobile Internet", International Journal of E-Business Research (IJEBR), vol. 10, no. 1, (2014), pp. 53-73.

[6] K. D. Chang, J. L. Chen and H. C. Chao, "The Potential Cloud Application Model for Internet of Things-Case Study of Shopping Malls”, Intelligent Information Hiding and Multimedia Signal Processing (IIH-MSP), 2014 Tenth International Conference on. IEEE, (2014), pp. 954-957. 
[7] J. W. Lian, "Critical factors for cloud based e-invoice service adoption in Taiwan: An empirical study", International Journal of Information Management, vol. 35, no. 1, (2015), pp. 98-109.

[8] K Boes, D. Buhalis and A. Inversini, "Conceptualising Smart Tourism Destination Dimensions[M]//Information and Communication Technologies in Tourism", 2015. Springer International Publishing, (2015), pp. 391-403.

[9] L. Liu, M. Zhang and Y. Lin, "A Survey on Workflow Management and Scheduling in Cloud Computing", Cluster, Cloud and Grid Computing (CCGrid), 2014 14th IEEE/ACM International Symposium on. IEEE, (2014), pp. 837-846.

[10] T. D. Owusu and C. Hoffman, "The Personalization and Prediction Innovation of Mobile Recommender Systems", Issues in Information Systems, vol. 15, no. 2, (2014).

[11] J. P. Ma, S. Wei and R. Lin, "Designing Cloud Computing into Taipei City: A Pilot Study of the Service Design from Taipei Cloud”, Cross-Cultural Design. Springer International Publishing, (2014), pp. 688-695.

[12] S. H. Alfaresi and K. Hone, "The Intention to Use Mobile Digital Library Technology: A Focus Group Study in the United Arab Emirates", International Journal of Mobile Human Computer Interaction (IJMHCI), vol. 7, no. 2, (2015), pp. 23-42.

[13] Q. Gu and M. Guirguis, "Secure Mobile Cloud Computing and Security Issues", High Performance Cloud Auditing and Applications. Springer New York, (2014), pp. 65-90.

[14] O. U. O. Khan, K. Ahsan and A. Salam, "Assistive Technology For Night Blind Tourist: A Mobile Application Approach", International Journal of E-Services and Mobile Applications (IJESMA), vol. 6, no 4, (2014), pp. 43-65.

[15] H. J. Ding, "Traffic Flow Data Collection and Signal Control System Based on Internet of Things and Cloud Computing", Advanced Materials Research, (2014), 846, pp. 1608-1611.

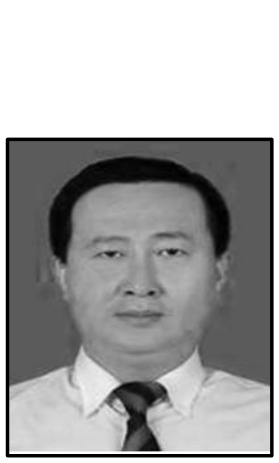

\section{Authors}

Lianlei Fan, received B.S. Mgt. Sci. in Tourism management from Xiangtan University in 2001, and M. Eng. in Project Management from Ocean University of China in 2011. He is currently working in Qingdao Vocational and Technical College of Hotel Management. His currenty research interests are on tourism management and project management.

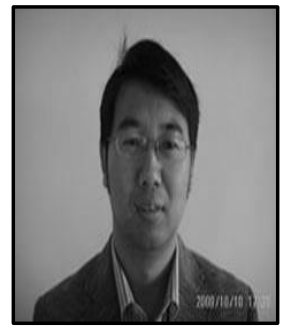

Shuguang Fu received the Bachelor degree in Computer Engineering from Qingdao Technological University and Master's degree in Computer Engineering from China Ocean University, CHINA in 2004 and 2011 respectively. He is teaching and researching Routing Protocols at Qingdao Vocational and Technical College of Hotel Management. 